\title{
Criterios de clasificación de las publicaciones científicas argentinas
}

\author{
Classification criteria of Argentine \\ scientific journals
}

Entre los días 11 y 12 de noviembre, tuvo lugar el Encuentro Iberoamericano de Editores Científicos 2010, organizado por el Centro Argentino de Información Científica y Tecnológica (Caicyt) conjuntamente con la Biblioteca Nacional, sede del evento.

En el marco del panel titulado "Incidencia de las publicaciones regionales en la evaluación científica", un grupo de editores propuso la firma de un escrito que planteaba la necesidad de repensar los criterios establecidos por las comisiones asesoras del Conicet para la evaluación de la producción científico-tecnológica de los postulantes a la Carrera de Investigador Científico.
Actualmente, cada comisión asesora establece sus propios criterios de validación y ponderación de los artículos según la "calidad" de las revistas en las que hayan sido publicados. Estos parámetros no están plasmados en un documento de carácter público, por lo que se desconocen los fundamentos utilizados por algunas de las comisiones para no asignar puntaje a los artículos publicados en revistas científicas argentinas, aunque cuenten con referato y estén indizadas en bases de datos internacionales.

A continuación transcribimos el cuerpo principal de la nota presentada a las autoridades del Conicet y la nómina de revistas e instituciones que respaldaron la propuesta.

Buenos Aires, 12/11/2010

Dra. Marta Rovira

Presidenta del CONICET

De nuestra mayor consideración:

Teniendo en cuenta que los trabajos científicos publicados en revistas nacionales de difusión internacional (que tienen referato y que están indizadas en bases de datos de prestigio internacional) no son consideradas para la evaluación por parte de las comisiones asesoras del CONICET, los abajo firmantes solicitan la revisión de este criterio discriminatorio hacia las revistas científicas de calidad editadas en Argentina.

Saludamos a usted muy atentamente

- Mathematicae Notae

- Revista Argentina de Microbiología

- Acta Bioquímica

- Clínica Latinoamericana

- Medicina (Buenos Aires)
- Pasado Por-venir

- Culturas

- La Alimentación Latinoamericana

- Cuadernos de Política Exterior Argentina

- Journal of Basic and Applied Genetics 
- La Zaranda de Ideas

- Revista de Arqueología Histórica Argentina y Latinoamericana

- Archivos de Pediatría

- Revista Médica del Uruguay

- American Journal of Sedimentology and Basin Analysis

- Ciencia, Docencia y Tecnología

- Latin American Applied Research

- Revista de la Unión Matemática Argentina

- Mat-SerieA

- Revista Escuela de Historia

- Boletín de la Sociedad Argentina de Botánica

- Salud Colectiva
- Revista de Enseñanza de la Física

- Arqueología

- Psiencia. Revista Latinoamericana de Ciencia Psicológica

- Darwiniana

- Estudios de Filosofía Práctica e Historia de las Ideas

- Revista de Geología Aplicada a la Ingeniería y al Ambiente

- Revistas de la Facultad de Humanidades y Ciencias de la Educación, Universidad Nacional de La Plata

- Revistas de la Facultad de Humanidades, Universidad Nacional de Mar del Plata

- Revistas de la Facultad de Filosofía y Letras, Universidad Nacional de Cuyo
Tal como se menciona en la última página de la nota, la temática fue tratada además en los paneles "Indicadores científicos, citas e índices: su incidencia en el posicionamiento de las publicaciones de la región" y "Diálogo entre editores".
El objetivo de publicar estas líneas es poder integrar a aquellos editores e investigadores, que no han estado presentes en el EIDEC 2010, a un diálogo que permita consensuar criterios entre los propios implicados, en pos de una mayor valoración de las publicaciones científicas argentinas.

\section{FORMA DE CITAR}

Criterios de clasificación de las publicaciones científicas argentinas. Salud Colectiva. 2010;6(3):371-372. 